\title{
Intra-abdominal infectious complications following gastrectomy in patients with excessive visceral fat
}

\author{
Norihiko Sugisawa $\cdot$ Masanori Tokunaga $\cdot$ \\ Yutaka Tanizawa $\cdot$ Etsuro Bando $\cdot$ \\ Taiichi Kawamura $\cdot$ Masanori Terashima
}

Received: 15 May 2011 / Accepted: 11 September 2011/Published online: 13 October 2011

(C) The International Gastric Cancer Association and The Japanese Gastric Cancer Association 2011

\begin{abstract}
Background Excessive visceral fat may be a better predictor of the development of postoperative morbidity after gastrectomy than body mass index (BMI). The aim of the present study was to clarify the most appropriate fat parameter to predict pancreas-related infection and anastomotic leakage following gastrectomy.

Methods The study was performed in 206 patients who underwent curative gastrectomy at the Shizuoka Cancer Center between April 2008 and March 2009. Relationships between fat parameters, including visceral fat area (VFA), and early surgical outcomes were investigated. The risk factors for pancreas-related infection and anastomotic leakage were identified using univariate and multivariate analyses.

Results There was no strong association between any of the fat parameters and operating time, intraoperative blood loss, the number of lymph nodes retrieved, or the duration of the postoperative hospital stay. Pancreas-related infection occurred in 18 patients $(8.7 \%)$, whereas anastomotic leakage was observed in 10 patients $(4.9 \%)$. Of all the fat parameters, only VFA was found to be an independent risk factor for both pancreas-related infection and anastomotic leakage, with odds ratios (95\% confidence intervals) of 1.015 (1.005-1.025) and 1.010 (1.000-1.021), respectively. Conclusions Excessive visceral fat, represented by the VFA, was found to be an independent risk factor for both pancreas-related infection and anastomotic leakage following gastrectomy.
\end{abstract}

\footnotetext{
N. Sugisawa $\cdot$ M. Tokunaga $\cdot$ Y. Tanizawa $\cdot$ E. Bando $\cdot$

T. Kawamura $\cdot$ M. Terashima $(\bowtie)$

Division of Gastric Surgery, Shizuoka Cancer Center,

1007 Shimonagakubo, Nagaizumi-cho, Sunto-gun,

Shizuoka 411-8777, Japan

e-mail: m.terashima@scchr.jp
}

Keywords Gastric cancer - Gastrectomy · Visceral fat . Postoperative complication

\section{Introduction}

Surgery is the only treatment strategy that offers the hope of a cure for gastric cancer patients. In Japan, in which the rates of gastric cancer are greater than those in Western countries, gastrectomy with D2 lymph node dissection is a well-established and widely accepted procedure [1, 2]. Although two large randomized controlled trials in Europe failed to demonstrate the efficacy of this procedure, due, in part, to increased postoperative morbidity and mortality [3, 4], recent reports suggest that gastrectomy with D2 lymph node dissection may be beneficial in certain patients [3-6]. One of the reasons for the unfavorable outcomes of gastrectomy with D2 lymph node dissection in the European studies may have been the higher proportion of obese patients in those studies.

Body mass index (BMI) is a simple index of weightfor-height that is commonly used to classify obesity. In Japan, the median BMI of gastric cancer patients, as well as that of the general population, has increased in recent years [7]. Although a relationship between BMI and postoperative morbidity has been reported previously, it remains contentious whether a high BMI is really associated with an increased rate of postoperative morbidity [8-12]. Recently, several reports have suggested that visceral fat area (VFA) is more strongly associated with postoperative intra-abdominal infectious complications, including pancreas-related infection and anastomotic leakage, than BMI $[13,14]$. However, this issue is also contentious. 
Therefore, in the present study we investigated the relationships between various fat parameters, including VFA, and early surgical outcomes following gastrectomy to clarify the most appropriate fat parameter to predict the development of pancreas-related infection and anastomotic leakage.

\section{Methods}

\section{Patients}

Between April 2008 and March 2009, a total of 217 patients underwent open distal or total gastrectomy with curative intent for primary gastric cancer at Shizuoka Cancer Center, Shizuoka, Japan. Patients who underwent splenectomy, distal pancreatic resection, and cholecystectomy were included in the study, whereas seven patients who underwent combined resection of other organs (liver, colon, and adrenal gland) and four patients who had synchronous cancer in other organs (colon, rectum, and kidney) were excluded from the study. Therefore, data from 206 patients were analyzed in the present study.

Lymph node station number, the degree of lymph node dissection, and pathological stage were determined on the basis of the Japanese classification of gastric carcinoma [15] and the Gastric cancer treatment guidelines in Japan [16]. Gastrectomy with D2 lymph node dissection was performed in patients with advanced gastric cancer, whereas D1 plus beta lymph node dissection (i.e., D1 + numbers 7, 8a, 9) was performed in patients with early gastric cancer.

\section{Fat measurement}

Multidetector computed tomography (MDCT) was performed in all patients prior to surgery. Patients were examined while in the supine position, with their arms stretched above their heads, at the end of inspiration, using a CT scanner (Aquilion; Toshiba Medical Systems, Tokyo, Japan). Parameters for scanning were: tube voltage, $120 \mathrm{kVp}$; scan time, $0.5 \mathrm{~s}$; and reconstruction slice thickness, $2 \mathrm{~mm}$. The tube current was determined automatically by the CT automatic exposure control system. The images obtained were transferred to a Ziostation workstation (Ziosoft, Tokyo, Japan), which was used to quantify the total fat area (TFA), subcutaneous fat area (SFA), and VFA at the level of the umbilicus. In the present study, relationships between early surgical outcomes following gastrectomy and TFA, VFA, SFA, and BMI, as the fat parameters, were investigated.

\section{Definition of early surgical outcomes}

Operating time, intraoperative blood loss, the number of lymph nodes retrieved, postoperative morbidity and mortality, and the duration of the postoperative hospital stay were investigated as early surgical outcomes.

Patients with Grade II or greater complications based on the Clavien-Dindo classification were defined as having postoperative morbidity [17]. In the present study, pancreas-related infection was diagnosed on the basis of the definitions of the International Study Group on Pancreatic Fistula (ISGPF) [18]. Diagnosis of anastomotic leakage was confirmed by radiological examination using contrast media. Postoperative mortality was defined as any death within 30 postoperative days (PODs).

\section{Statistical analysis}

Spearman's rank correlation coefficient was used to evaluate relationships between individual fat parameters (BMI, TFA, SFA, and VFA) and early surgical outcomes (operating time, intraoperative blood loss, number of lymph nodes retrieved, postoperative hospital stay). Relationships between individual fat parameters and the categorical variables of pancreas-related infection and anastomotic leakage were evaluated using logistic regression.

To identify independent risk factors for each of the intra-abdominal infectious complications, a multivariate logistic regression model with forward selection was used, with BMI, TFA, SFA, VFA, age, sex, operating time, intraoperative blood loss, number of lymph nodes retrieved, surgical procedure (total or distal gastrectomy), type of lymph node dissection (D2 or D1 plus beta), and splenectomy (yes or no) included as covariates.

All statistical analyses were performed using SPSS version 13.0 (SPSS, Chicago, IL, USA). All continuous data are presented as medians (ranges). $P<0.05$ was considered significant.

\section{Results}

Patients' characteristics

The patients' characteristics are given in Table 1. Total gastrectomy was performed in 48 patients, 29 of whom also underwent splenectomy for the removal of splenic hilar lymph nodes. Additional pancreatosplenectomy was performed in 2 of the 48 patients. The remaining 158 patients underwent distal gastrectomy. D2 lymph node dissection was performed in 111 patients, whereas 95 patients underwent D1 plus beta lymph node dissection.

Table 2 lists the early surgical outcomes of all patients. Postoperative complications were observed in 55 patients (26.7\%). The incidence of pancreas-related infection and anastomotic leakage was $8.7 \%$ and $4.9 \%$, respectively. Five patients had both pancreas-related infection and 
Table 1 Clinocopathological characteristics of patients

\begin{tabular}{ll}
\hline Age (years) & $65.9(39-89)$ \\
Sex (male/female) & $146 / 60$ \\
Diabetes mellitus $(n)$ & 17 \\
Individual fat parameter & \\
BMI $\left(\mathrm{kg} / \mathrm{m}^{2}\right)$ & $23(16.0-32.3)$ \\
TFA $\left(\mathrm{cm}^{2}\right)$ & $198(6.9-505.8)$ \\
SFA $\left(\mathrm{cm}^{2}\right)$ & $107.4(3.0-266.9)$ \\
VFA $\left(\mathrm{cm}^{2}\right)$ & $90.5(3.6-262.5)$ \\
Surgical procedure $(n)$ & \\
Total gastrectomy & 48 \\
Distal gastrectomy & 158 \\
Lymph node dissection $(n)$ & \\
D2 & 111 \\
D1 plus beta & 95 \\
Splenectomy $(n)$ & \\
Yes & 29 \\
No & 177 \\
Pathological stage $(n)$ & \\
IA & 88 \\
IB & 48 \\
II & 44 \\
IIIA & 15 \\
IIIB & 9 \\
IV & 2 \\
\hline
\end{tabular}

Unless indicated otherwise, values are means, with ranges given in parentheses

$B M I$ body mass index, $T F A$ total fat area, $S F A$ subcutaneous fat area, $V F A$ visceral fat area

Table 2 Early surgical outcomes of 206 patients

\begin{tabular}{ll}
\hline Operating time (min) & $194(103-489)$ \\
Intraoperative blood loss (mL) & $265(13-2606)$ \\
No. of lymph nodes retrieved & $37(8-109)$ \\
Postoperative complications & $55(26.7 \%)$ \\
$\quad$ Pancreas-related infection & $18(8.7 \%)$ \\
$\quad$ Anastomotic leakage & $10(4.9 \%)$ \\
Postoperative hospital deaths & $1(0.5 \%)$ \\
Duration of postoperative hospital stay (days) & $11(7-87)$ \\
\hline
\end{tabular}

Values are presented as either median with ranges in parentheses or as the number of patients in each group, with percentages in parentheses

anastomotic leakage. Both of the two patients who underwent distal pancreatectomy had pancreas-related infection. Postoperative mortality was observed in one patient $(0.5 \%)$. This patient had undergone distal gastrectomy with D2 lymph node dissection, and anastomotic leakage developed on POD 7. This patient died suddenly on POD 9 due to a pulmonary embolism.
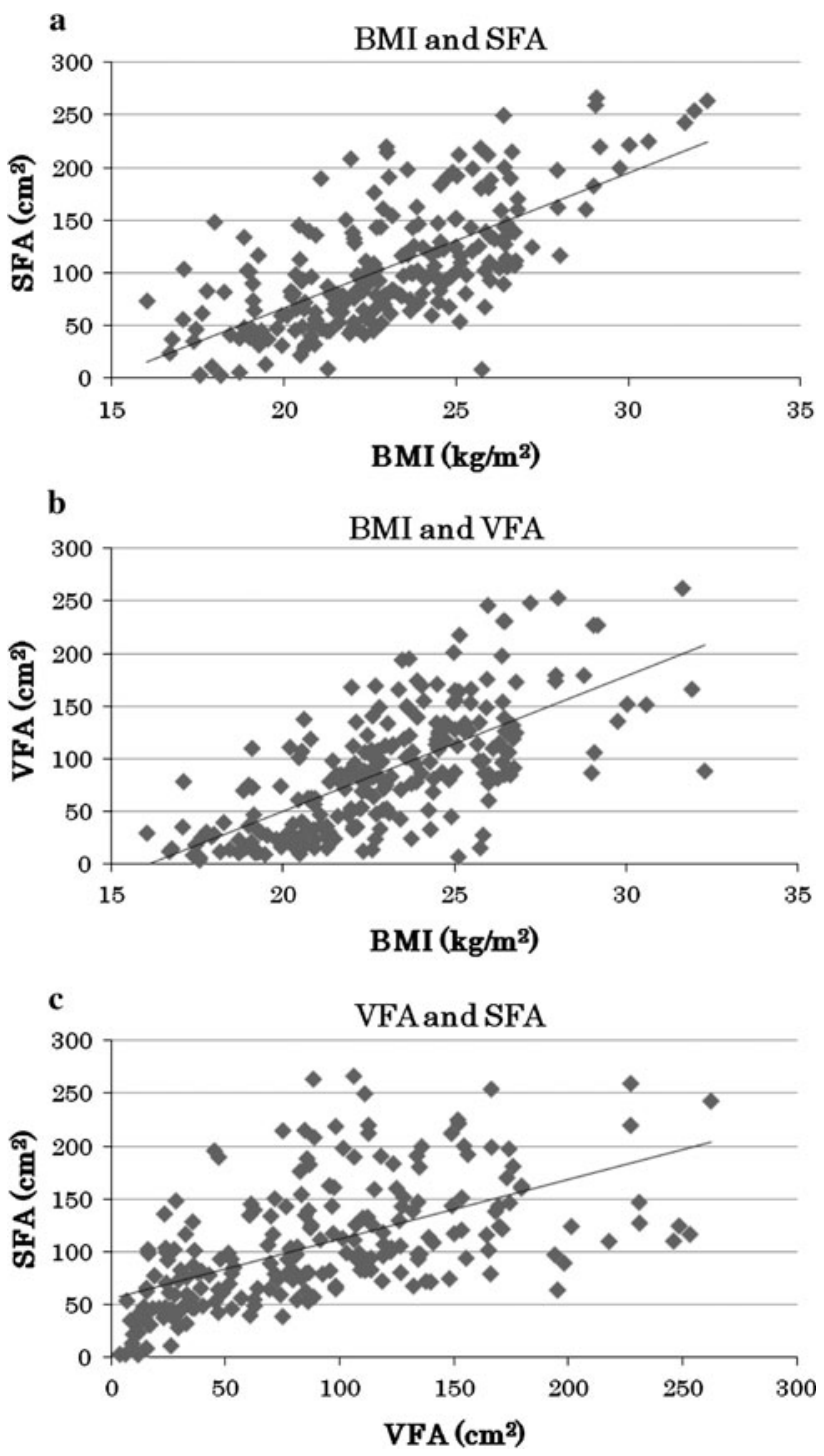

Fig. 1 Correlations between a superficial fat area (SFA) and body mass index $(B M I) ; R=0.672, P<0.001$, b visceral fat area $(V F A)$ and BMI; $R=0.683, P<0.001$, and $\mathbf{c}$ SFA and VFA; $R=0.555$, $P<0.001$. Significant associations were observed for all comparisons

Relationships between fat parameters and early surgical outcomes

Figure 1 shows the correlations between SFA, VFA, and BMI. Significant correlations were found between SFA and both BMI and VFA, as well as between VFA and BMI. Correlation coefficients for each of the fat parameters and operating time, intraoperative blood loss, number of lymph nodes retrieved, and postoperative hospital stay are given in Table 3. Although VFA was weakly associated with prolonged operating time (correlation coefficient 0.304) and increased intraoperative blood loss (correlation coefficient 0.371 ), no significant relationships were observed for any of the fat parameters and operation time, 
Table 3 Relationship between fat parameters and early surgical outcome data

\begin{tabular}{lllll}
\hline & Intraoperative blood loss & Operating time & No. of lymph nodes retrieved & Postoperative hospital stay \\
\hline BMI & $0.295(<0.001)$ & $0.235(0.001)$ & $-0.196(0.005)$ & $0.011(0.872)$ \\
TFA & $0.322(<0.001)$ & $0.250(<0.001)$ & $-0.134(0.055)$ & $0.103(0.139)$ \\
SFA & $0.199(0.004)$ & $0.153(0.028)$ & $-0.022(0.756)$ & $0.025(0.726)$ \\
VFA & $0.371(<0.001)$ & $0.304(<0.001)$ & $-0.197(0.005)$ & $0.155(0.026)$ \\
\hline
\end{tabular}

Values are the correlation coefficients, with $P$ values given in parentheses

$B M I$ body mass index, TFA total fat area, $S F A$ subcutaneous fat area, $V F A$ visceral fat area

Table 4 Identification of risk factors for the development of pancreas-related infection and anastomotic leakage, determined using univariate analysis

\begin{tabular}{|c|c|c|c|c|c|c|}
\hline & \multicolumn{3}{|c|}{ Pancreas-related infection } & \multicolumn{3}{|c|}{ Anastomotic leakage } \\
\hline & Odds ratio & $95 \% \mathrm{CI}$ & $P$ & Odds ratio & $95 \% \mathrm{CI}$ & $P$ \\
\hline BMI $\left(\mathrm{kg} / \mathrm{m}^{2}\right)$ & 1.318 & $1.121-1.548$ & 0.001 & 1.156 & $0.946-1.411$ & 0.156 \\
\hline TFA $\left(\mathrm{cm}^{2}\right)$ & 1.009 & $1.004-1.014$ & 0.001 & 1.003 & $0.997-1.009$ & 0.291 \\
\hline SFA $\left(\mathrm{cm}^{2}\right)$ & 1.008 & $1.001-1.016$ & 0.035 & 0.999 & $0.987-1.010$ & 0.802 \\
\hline VFA $\left(\mathrm{cm}^{2}\right)$ & 1.016 & $1.008-1.025$ & 0.001 & 1.010 & $1.000-1.021$ & 0.042 \\
\hline Age (years) & 0.978 & $0.934-1.023$ & 0.332 & 0.997 & $0.937-1.061$ & 0.923 \\
\hline Sex (male or female) & 2.335 & $0.655-8.323$ & 0.191 & 1.681 & $0.346-8.158$ & 0.519 \\
\hline Intraoperative blood loss (mL) & 1.002 & $1.001-1.003$ & 0.001 & 1.001 & $1.000-1.002$ & 0.227 \\
\hline Operating time (min) & 1.010 & $1.003-1.018$ & 0.007 & 1.006 & $0.996-1.016$ & 0.234 \\
\hline No. of lymph nodes retrieved & 0.987 & $0.954-1.021$ & 0.458 & 0.961 & $0.912-1.012$ & 0.133 \\
\hline Surgical procedure (total or distal) & 5.574 & $2.094-14.841$ & 0.001 & 2.303 & $0.622-8.526$ & 0.212 \\
\hline Lymph node dissection (D2 or D1) & 3.555 & $1.137-11.110$ & 0.029 & 1.300 & $0.356-4.751$ & 0.692 \\
\hline Splenectomy (yes or no) & 7.515 & 2.729-20.694 & 0.001 & 0.667 & $0.081-5.468$ & 0.706 \\
\hline
\end{tabular}

$C I$ confidence interval, $B M I$ body mass index, $T F A$ total fat area, $S F A$ subcutaneous fat area, $V F A$ visceral fat area

intraoperative blood loss, the number of lymph nodes retrieved, or the duration of the postoperative hospital stay.

Risk factors for intra-abdominal infectious complications

Tables 4 and 5 list the results of univariate and multivariate analyses used to identify risk factors for intra-abdominal infectious complications. On the basis of the univariate analysis, all fat parameters, operating time, intraoperative blood loss, surgical procedure, type of lymph node dissection, and splenectomy affected the development of pancreas-related infection. Multivariate analysis revealed that VFA, intraoperative blood loss, and splenectomy were independent risk factors for pancreas-related infection, with odds ratios (95\% confidence intervals) of 1.015 (1.005-1.025), 1.001 (1.000-1.003), and 7.125 (2.08324.372), respectively. With regard to anastomotic leakage, both univariate and multivariate analyses revealed VFA as a risk factor for the development of anastomotic leakage, with an odds ratio (95\% confidence interval) on multivariate analysis of $1.010(1.000-1.021)$.
Table 5 Multivariate analysis identification of independent risk factors for the development of pancreas-related infection

\begin{tabular}{llll}
\hline & Odds ratio & $95 \%$ CI & $P$ \\
\hline VFA $\left(\mathrm{cm}^{2}\right)$ & 1.015 & $1.005-1.025$ & 0.004 \\
Intraoperative blood loss $(\mathrm{mL})$ & 1.001 & $1.000-1.003$ & 0.009 \\
Splenectomy (yes or no) & 7.125 & $2.083-24.372$ & 0.002 \\
\hline
\end{tabular}

$C I$ confidence interval, $V F A$ visceral fat area

In order to justify the use of correlation analysis to find risk factors for the surgical complications, it was mandatory to prove that the fat components did not relate to outcomes in binomial fashion. To do so, we divided the patients into 4 groups according to the VFA $(<35.8$, $35.8-85.6,85.6-126.5$, and $>126.5 \mathrm{~cm}^{2}$ ), and looked at the incidence of surgical complications in each group. Pancreas-related complications were observed in $0,2,8$, and 8 patients, respectively, in these 4 groups, showing that the relationship between VFA and surgical complications was not binomial. 


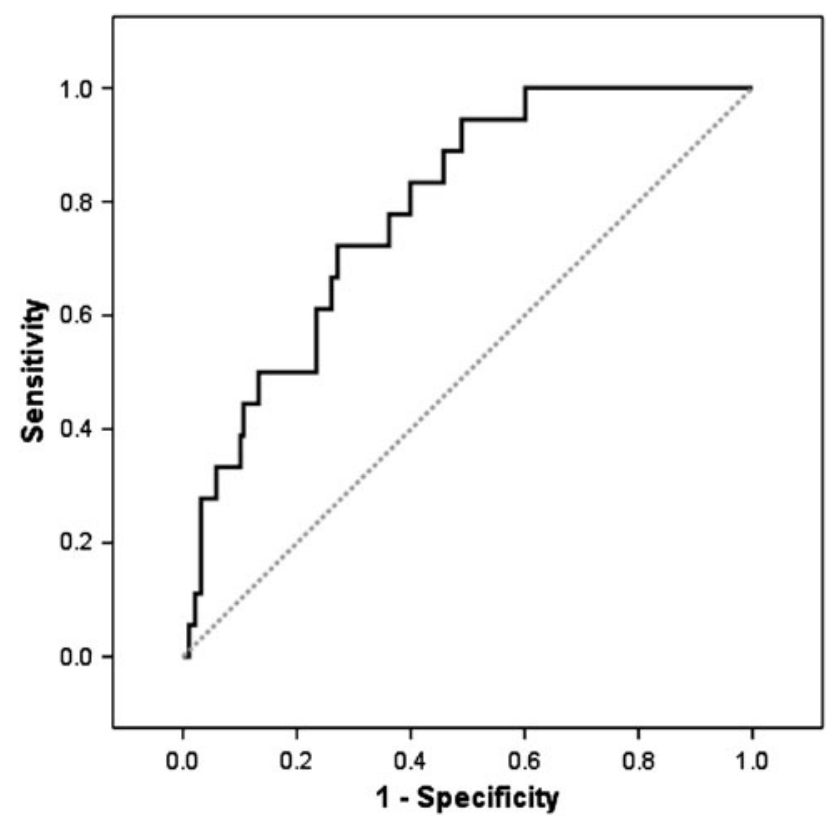

Fig. 2 Receiver operating characteristic (ROC) curve to identify the appropriate cut-off value of VFA to predict pancreas-related infection. The area under the curve (AUC) was 0.787 and the threshold of VFA was $113.6 \mathrm{~cm}^{3}$ with sensitivity of $72.2 \%$ and specificity of $62.9 \%$

The cut-off value for VFA as an indicator of pancreas-related infection

Figure 2 shows the receiver operating characteristic (ROC) curve used to identify the appropriate cut-off value of VFA to predict pancreas-related infection. The area under the curve (AUC) was 0.787 and the threshold of VFA was $113.6 \mathrm{~cm}^{2}$ with sensitivity of $72.2 \%$ and specificity of $62.9 \%$.

\section{Discussion}

The incidence of postoperative morbidity following gastrectomy with lymph node dissection (D2 or more) has been reported to be $17-21 \%$ in eastern Asia $[19,20]$ and $21-46 \%$ in Europe [3, 4, 21-25]. Previous studies of the risk factors for postoperative morbidity indicate that obesity, defined as BMI $>25 \mathrm{~kg} / \mathrm{m}^{2}$, is one of the most important [8-10]. The recent development of specific computer software has enabled the easy calculation of the amount of visceral fat, and some authors have suggested that the VFA may be a better predictor of the development of postoperative morbidity than the BMI [13, 14].

Of all morbidities, intra-abdominal infectious complications, including pancreas-related infection and anastomotic leakage, are potentially fatal complications; thus, in the present study, we investigated independent risk factors for both of these complications. Although Tokunaga et al. [13] have reported that excessive visceral fat is a risk factor for postoperative intra-abdominal infectious complications and Tanaka et al. [14] have reported that the amount of visceral fat affects the development of pancreas-related infection, independent risk factors for both complications had not been investigated simultaneously in previous studies. In the present study, we determined the factors affecting the development of both pancreas-related infection and anastomotic leakage.

The results of the present study indicate that a high VFA is associated with the development of both pancreas-related infection and anastomotic leakage following gastrectomy. To date, the risk factors for anastomotic leakage after gastrectomy have not been completely clarified [26]. Both Ser et al. [27] and Kang et al. [28] have reported that anastomotic leakage may occur in cases in which there is excess tension and pressure on the anastomotic site and that these conditions are more frequently observed in patients with excessive visceral fat because the thick mesentery creates tension on the anastomosis. In addition, a deeper surgical field in these patients, and preoperative comorbidities, such as cardiovascular disease or diabetes mellitus, which are frequently seen in obese patients, may affect the development of anastomotic leakage [29-31].

In the present study, pancreas-related infections were observed in 18 patients $(8.7 \%$ ), with splenectomy, intraoperative blood loss, and VFA identified as independent risk factors. Splenectomy is a well-known and widely accepted risk factor, because manipulation of the tail of the pancreas during the procedure increases the risk of pancreas-related infection $[6,14,31]$. In Europe, the final results of the Dutch D1D2 trial recommended D2 gastrectomy. However, they also recommended that the spleen should be preserved, because of increased morbidity and mortality after splenectomy [32]. In Japan, though the current standard treatment for upper-third gastric cancer is a total gastrectomy with splenectomy, a recent randomized controlled trial revealed a high incidence of postoperative complications after splenectomy [33]. We should await final survival analysis of this study before we conclude whether or not the spleen has to be preserved. Distal pancreatectomy has been thought to be correlated with pancreas-related complications. In the present study, actually, both of the two patients with pancreatectomy had this complication. However, the number was so small that further analysis could not be done.

In the present study, excessive visceral fat also increased the incidence of pancreas-related infection. It has been proposed that excessive visceral fat makes it difficult to find the border between the pancreas and lymph nodes, which may result in intraoperative pancreatic injury [13, 14]. Our ROC analysis revealed that a VFA of $113.6 \mathrm{~cm}^{2}$ 
was an appropriate cut-off value. Careful surgery will be required particularly in these patients having a VFA of $113.6 \mathrm{~cm}^{2}$ or more.

Although the present study identified a significant relationship between intraoperative blood loss and pancreasrelated infection, others have reported that increased bleeding does not affect the incidence of pancreas-related infection $[34,35]$. We believe that increased bleeding may have created difficulties in identifying the border between the pancreas and lymph nodes, as occurs in patients with excessive visceral fat, thus contributing to an increased incidence of pancreas-related infection.

Preoperative co-morbidities have also been considered to affect the incidence of postoperative complications. Also, poor nutritional status due to advanced primary gastric cancer may be associated with a high incidence of postoperative complications. However, in the present study, the patients' preoperative nutritional status (performance status, serum albumin level) and co-morbidities (diabetes mellitus, hypertension) were not associated with the incidence of intra-abdominal infectious complications (data not shown).

In conclusion, excessive visceral fat, represented by the VFA, was found to be an independent risk factor for both pancreas-related infection and anastomotic leakage following gastrectomy. Greater diligence during surgery is necessary for patients with excessive visceral fat, particularly if splenectomy has to be performed simultaneously.

\section{References}

1. Maruyama K, Okabayashi K, Kinoshita T. Progress in gastric cancer surgery in Japan and its limits of radicality. World J Surg. 1987;11:418-25.

2. Sasako M, McCulloch P, Kinoshita T, Maruyama K. New method to evaluate the therapeutic value of lymph node dissection for advanced gastric cancer. Br J Surg. 1995;82:346-51.

3. Bonenkamp JJ, Songun I, Hermans J, Sasako M, Welvaart K, Plukker JTM, et al. Randomised comparison of morbidity after D1 and D2 dissection for gastric cancer in 996 Dutch patients. Lancet. 1995;2345:745-8.

4. Cuschieri A, Fayers P, Fielding J, Craven J, Bancewicz J, Joypaul $\mathrm{V}$, et al. Postoperative morbidity and mortality after D1 and D2 resections for gastric cancer: preliminary results of the MRC randomised controlled surgical trial. Lancet. 1996;347:995-9.

5. Bonekamp JJ, Hermans J, Sasako M, van de Velde CJH. Extend lymph-node dissection for gastric cancer. $N$ Engl J Med. 1999;340:908-14.

6. Cuschieri A, Weeden S, Fielding J, Bancewicz J, Craven J, Joypaul V, et al. Patient survival after D1 and D2 resections for gastric cancer: long-term results of the MRC randomized surgical trial. Surgical Co-operative Group. Br J Cancer. 1999;79: 1522-30.

7. Kubo M, Sano T, Fukagawa T, Katai H, Sasako M. Increasing body mass index in Japanese patients with gastric cancer. Gastric Cancer. 2005;8:39-41.
8. Inagawa S, Adachi S, Oda T, Kawamoto T, Koike N, Fukao K. Effect of fat volume on postoperative complications and survival rate after D2 dissection for gastric cancer. Gastric Cancer. 2000;3:141-4.

9. Tsujinaka T, Sasako M, Yamamoto S, Sano T, Kurokawa Y, Nashimoto A, et al. Influence of overweight on surgical complications for gastric cancer: results from a randomized control trial comparing D2 and extended paraaortic D3 lymphadenectomy (JCOG9501). Ann Surg Oncol. 2007;14:355-61.

10. Ojima T, Iwahashi M, Nakamori M, Nakamura M, Naka T, Ishida $\mathrm{K}$, et al. Influence of overweight on patients with gastric cancer after undergoing curative gastrectomy. Arch Surg. 2009;144(4): $351-8$.

11. Gretschel S, Christoph F, Bembenek A, Estevez-Schwarz L, Schneider U, Schlag PM. Body mass index does not affect systematic D2 lymph node dissection and postoperative morbidity in gastric cancer patients. Ann Surg Oncol. 2003;10:363-8.

12. Lee JH, Paik YH, Lee JS, Ryu KW, Kim CG, Park SR, et al. Abdominal shape of gastric cancer patients influences short-term surgical outcomes. Ann Surg Oncol. 2007;14:1288-94.

13. Tokunaga M, Hiki N, Fukunaga T, Ogura T, Miyata S, Yamaguchi T. Effect of individual fat areas on early surgical outcomes after open gastrectomy for gastric cancer. Br J Surg. 2009; 96:496-500.

14. Tanaka K, Miyashiro I, Yano M, Kishi K, Motoori M, Seki Y, et al. Accumulation of excess visceral fat is a risk factor for pancreatic fistula formation after total gastrectomy. Ann Surg Oncol. 2009;16:1520-5.

15. Japanese Gastric Cancer Association. Japanese classification of gastric carcinoma-2nd English edition. Gastric Cancer. 1998;1: $10-24$.

16. Nakajima T. Gastric cancer treatment guidelines in Japan. Gastric Cancer. 2002;5:1-5.

17. Dindo D, Demartines N, Clavien PA. Classification of surgical complications. A new proposal with evaluation in a cohort of 6336 patients and results of a survey. Ann Surg. 2004;240: 205-13.

18. Bassi C, Dervenis C, Butturini G, Fingerhut A, Yeo C, Izbicki J, et al. Postoperative pancreatic fistula: an international study group (ISGPF) definition. Surgery. 2005;138:8-13.

19. Sano T, Sasako M, Yamamoto S, Nashimoto A, Kurita A, Hiratsuka $\mathrm{M}$, et al. Gastric cancer surgery: morbidity and mortality results from a prospective randomized controlled trial comparing D2 and extended para-aortic lymphadenectomy-Japan Clinical Oncology Group Study 9501. J Clin Oncol. 2004;22:2767-73.

20. Wu CW, Hsiung CA, Lo SS, Hsieh MC, Shia LT, Whang-Peng J. Randomized clinical trial of morbidity after D1 and D3 surgery for gastric cancer. Br J Surg. 2004;91:283-7.

21. de Manzoni G, Verlato G, Guglielmi A, Laterza E, Genna M, Cordiano C. Prognostic significance of lymph node dissection in gastric cancer. Br J Surg. 1996;83:1604-7.

22. Smith BR, Stabile BE. Aggressive D2 lymphadenectomy is required for accurate pathologic staging of gastric adenocarcinoma. Am Surg. 2006;72:849-52.

23. Marrelli D, Pedrazzani C, Neri A, Corso G, DeStefano A, Pinto E, et al. Complications after extended (D2) and superextended (D3) lymphadenectomy for gastric cancer: analysis of potential risk factors. Ann Surg Oncol. 2007;14:25-33.

24. Danielson H, Kokkola A, Kiviluoto T, Siren J, Luohimo J, Kivilaakso E, et al. Clinical outcome after D1 vs D2-3 gastrectomy for treatment of gastric cancer. Scand J Surg. 2007;96:35-40.

25. Degiuli M, Sasako M, Ponti A, Calvo F. Survival results of a multicentre phase II study to evaluate D2 gastrectomy for gastric cancer. Br J Cancer. 2004;90:1727-32.

26. Sierzega M, Kolodziejczyk J, Kulig J, Polish Gastric Cancer Study Group. Impact of anastomotic leakage on long-term 
survival after total gastrectomy for carcinoma of the stomach. $\mathrm{Br}$ J Surg. 2010;97:1035-42.

27. Ser $\mathrm{KH}$, Lee WJ, Lee YC, et al. Experience in laparoscopic sleeve gastrectomy for morbidly obese Taiwanese: staple-line reinforcement is important for preventing leakage. Surg Endosc. 2010;24:2253-9.

28. Kang KC, Cho GS, Han SU, Kim W, Kim HH, Kim MC, et al. Comparison of Billroth I and Billroth II reconstructions after laparoscopy-assisted distal gastrectomy: a retrospective analysis of large-scale multicenter results from Korea. Surg Endosc. 2011;25:1953-61.

29. Grotenhuis BA, Wijnhoven BPL, Hötte GJ, van der Stok EP, Tilanus HW, van Lanshot JJB, et al. Prognostic value of body mass index on short-term and long-term outcome after resection of esophageal cancer. World J Surg. 2010;34:2621-7.

30. Pi-Sunyer FX. Medical hazards of obesity. Ann Intern Med. 1993;119:655-60.
31. Despres JP, Lemieux I. Abdominal obesity and metabolic syndrome. Nature. 2006;444:881-7.

32. Songun I, Putter H, Kranenbarg EM, Sasako M, Van de Velde CJ. Surgical treatment of gastric cancer: 15-year follow-up results of the randomised nationwide Dutch D1D2 trial. Lancet Oncol. 2010;11:439-49.

33. Sano T, Sasako M, Shibata T, Yamamoto S, Tsuburaya A, Nashimoto A, et al. Randomized controlled trial to evaluate splenectomy in total gastrectomy for proximal gastric carcinoma (JCOG0110): Analyses of operative morbidity, operation time, and blood loss. J Clin Oncol 2010;28:15s (suppl; abstr 4020).

34. Katai H, Yoshimura K, Fukagawa T, Sano T, Sasako M. Risk factors for pancreas-related abscess after gastrectomy. Gastric Cancer. 2005;8:137-41.

35. Nobuoka D, Gothda N, Konishi M, Nakagohri T, Takahashi S, Kinoshita T. Prevention of postoperative pancreatic fistula after total gastrectomy. World J Surg. 2008;32:2261-6. 